\title{
Treatment outcomes of intrauterine insemination and pregnancy prognosis factors: a retrospective single-center cohort study
}

\begin{abstract}
Although intrauterine insemination (IUI) utilizing the husband's semen is widely used as a general fertility treatment, there are no clear policies determining the appropriate number of IUI cycles or a selection criteria for couples at our institution. In this study we investigated IUIs performed at our facilities in the past 3 years, and examined prognostic factors for pregnancy based on age, number of inseminations, and semen findings. A total of 341 patients who underwent 1,229 cycles of IUIs performed at our institution between January 2016 and December 2018 were included. The pregnancy rate was $6.2 \%$ per cycle and $21.1 \%$ per couple. In terms of age, we noted both men and women contributed to a decrease in the pregnancy rate with increasing age, but the effects of aging tended to be slightly less pronounced in men. The cumulative pregnancy rate for the number of times insemination was performed was approximately $50 \%$ by the second cycle, and $90 \%$ by the sixth. Assessment of semen analysis showed significant differences in motility and adjusted recovered sperm concentration $(\mathrm{P}<0.05)$.
\end{abstract}

Keywords: intrauterine insemination, pregnancy outcome, semen analysis
Volume 4 Issue 3 - 2020

\author{
Hiroki Onoue,Tsukasa Baba, Kanako Koiwa, \\ Michiko Kon, Shigeichiro Tsuchiya, Chie \\ Satoh \\ Department of Obstetrics and Gynecology, Iwate Medical \\ University School of Medicine, Japan
}

\begin{abstract}
Correspondence: Hiroki Onoue, Department of Obstetrics and Gynecology, Iwate Medical University School of Medicine, Japan, Fax +8I-19-907-6749, Tel +8I-19-65I-5 III, Email onoue0720@gmail.com

Correspondence: Tsukasa Baba, Department of Obstetrics and Gynecology, Iwate Medical University School of Medicine, Japan, Fax +8I- 19-907-6749, Tel +8I-19-65I-5III, Email babatsu@iwate-med.ac.jp
\end{abstract}

Received: May 07, 2020 | Published: May 18, 2020
Abbreviations: IUI, although intrauterine insemination; IVF, in vitro fertilization

\section{Introduction}

Intrauterine insemination (IUI) using the husband's semen, is a treatment for infertility due to mild male factors, sexual intercourse problems, and in cases of unexplained infertility where regular intercourse does not result in pregnancy after a certain time period. In recent years, the average age of marriage in Japan has risen, and the age at which couples who visit our hospital to have a baby is also rising. The couples who come for treatment want to be pregnant in the shortest time possible, and the therapist performing the IUI needs to, as far as possible, accurately predict when pregnancy will occur, to advise how long to continue the IUI, and at what point to step up to in vitro fertilization (IVF). There are several factors reported to affect pregnancy rates with IUI. For women, age, mature follicle count and endometrial thickness, ${ }^{1}$ and in men, sperm concentration and total motile sperm count $t^{1,2}$ are predictive of pregnancy. We perform IUIs for patients without bilateral tubal obstruction or severe male factor infertility, without any age restrictions. In this study, we summarized the outcomes of IUIs performed at our hospital over the past 3 years and examined whether age and semen findings could be predictive of pregnancy rates.

\section{Materials and methods}

\section{Study subjects}

This retrospective study of couples undergoing IUI was carried out at Iwate Medical University, between January 2016 and December 2018. This study was conducted in compliance with the Declaration of Helsinki and according to the Ethical Guidelines for Medical and Health Research Involving Human Subjects established by the Ministry of Health, Labour, and Welfare in Japan. The protocol of registry for assisted reproduction was approved by the ethical review committee of the Iwate Medical University Hospital (MH2018-527).

\section{Methods}

For cases with no ovulation disorders the IUIs were performed during the natural cycle, while those with ovulation disorders the IUIs were performed during a regulated ovarian stimulation cycle. For ovarian stimulation, clomiphene citrate (Clomid ${ }^{\circledR}$ Fuji Pharmaceutical Industry Tokyo, Japan), cyclophenyl (Sekisovit ${ }^{\circledR}$ Asuka Pharmaceutical Tokyo, Japan), HMG (HMG for intramuscular injection ${ }^{\circledR}$ Fuji Pharmaceutical Tokyo, Japan), and recombinant FSH (Gonal F® Merck Biopharma Co., Ltd. Tokyo, Japan) were used alone or in combination as appropriate. Follicles were measured by transvaginal ultrasonography a few days before ovulation was predicted, and IUI was planned if the chief follicle was greater than $18 \mathrm{~mm}$. On the day of IUI or the day before, hCG was administered at 5000 IU (HCG Mochida Muscle Injection ${ }^{\circledR}$ Mochida Pharmaceutical Tokyo Japan).

\section{Semen analysis and sperm preparation}

Semen was collected in the hospital's semen collection room or brought in within 2 hours of home collection. After liquefaction, sperm were measured using a Maclar sperm analysis counting chamber. Sperm adjustment was performed using the density gradient method. The liquefied semen was layered in $3 \mathrm{ml}$ of total Isolate $\mathbb{R}$ (Irvine Scientific, California, USA) and centrifuged at $3000 \mathrm{rpm}$ for $10 \mathrm{~min}$. The supernatant was discarded, and the bottom sperm 
sediment aspirated and suspended in $4 \mathrm{ml}$ of $\mathrm{mHTF}$ (Irvine Scientific, California, USA). A second centrifugation was then performed (1200 rpm, $5 \mathrm{~min}$ ), the supernatant discarded, and the sperm sediment suspended in $1 \mathrm{ml}$ of $\mathrm{mHTF}$.

\section{Intrauterine insemination.}

IUI was performed using a $1 \mathrm{ml}$ syringe and a soft AIH catheter (Soft Medical Inc., Tokyo, Japan) after confirming the shape of the uterus by transvaginal ultrasonography.

\section{Statistical analysis}

Results were expressed as mean \pm standard deviation. The Mann-Whitney $U$ test for nonparametric variables was used for statistical analysis. The $\chi$-square test was used for the semen analysis. Spearman's rank correction coefficients and linear regression lines were calculated for the female and male ages. $\mathrm{P}<0.05$ was considered statistically significant.

\section{Results}

1,229 IUI cycles (341 couples) were performed. The mean age of the women and men at the time of IUI was $36.3 \pm 4.7$ years and $38.3 \pm 5.9$ years respectively (Table 1 ). There were 76 cycles that resulted in pregnancy. The pregnancy rate per cycle was $6.2 \%$ and the pregnancy rate per case was $21.1 \%$. The mean ages of the women and men in cases of pregnancy were $34.0 \pm 4.4$ and $36.5 \pm 5.5$ years respectively. The mean ages of women and men in non-pregnant cases were $36.5 \pm 4.7$ and $38.4 \pm 6.8$ years respectively (Table 2 ). We examined the number of IUI cycles and pregnancy rates for couples of every age group Figure $1 \& 2$ at 3 -year intervals. The number of treatment cycles peaked at the age of 35-37 years for women and $38-40$ years for men. The pregnancy rate was highest at $11.1 \%$ for women 26-28 years of age, ranged from 5-10\% until 40 years of age, but declined markedly after 41 years of age. On the other hand, when looking at the age of men, it was the highest at $20 \%$ in the age group of 23-25 years, and it was 5-10\% before 46 years of age, with a marked decline after 47 years of age. In both the pregnant and non-pregnant cases, the age of the couples positively correlated with each other (Figure 3). The first IUI was the most successful, achieving pregnancy in $32.9 \%$ of the cases, $17.1 \%$ by the third time, and $3.9 \%$ by the fifth time (Figure 4). The semen analyses on the day of insemination were compared between pregnant and non-pregnant cycles. Significant differences were found in motility and concentration of recovered sperm $(\mathrm{P}<0.05)$ (Table 3$)$. The lowest values for each parameter in the pregnancy cases were $0.5 \mathrm{ml}$ of the original semen volume, $5 \times 10^{6} / \mathrm{ml}$ of concentration, and $0.3 \times 10^{6} / \mathrm{ml}$ concentration of recovered sperm, and $15 \%$ motility (Table 4 ).

Table I Summary of cases

\begin{tabular}{ll}
\hline Summary of cases & \\
\hline Number of cycles & 1229 \\
Number of couples & 341 \\
Female age & $36.3 \pm 4.7(23-46)$ \\
Male age & $38.3 \pm 5.9(24-59)$ \\
\hline
\end{tabular}

Table 2 Pregnant and non-pregnant cases

\begin{tabular}{llll}
\hline & Pregnancy & Non-pregnancy & P value \\
\hline Number of cycles & 76 & 1153 & \\
Female age & $34.0 \pm 4.4(23-44)$ & $36.5 \pm 4.7(23-46)$ & $<0.0001$ \\
Male age & $36.5 \pm 5.5(24-49)$ & $38.4 \pm 6.8(25-59)$ & $<0.0001$ \\
$\begin{array}{l}\text { Pregnancy rate } \\
\text { per cycle (\%) }\end{array}$ & 6.2 & & \\
$\begin{array}{l}\text { Pregnancy rate } \\
\text { per couple (\%) }\end{array}$ & 21.1 & & \\
\hline
\end{tabular}

Table 3 Comparison of semen analysis

\begin{tabular}{llll}
\hline & Pregnancy & $\begin{array}{l}\text { Non- } \\
\text { pregnancy }\end{array}$ & P value \\
\hline Volume $(\mathrm{ml})$ & $2.4 \pm 1.2$ & $2.6 \pm 1.0$ & 0.39 \\
Concentration $(\times 106 / \mathrm{ml})$ & $66.4 \pm 41.6$ & $63.6 \pm 46.6$ & 0.58 \\
& $52.9 \pm 14.8$ & $48.0 \pm 21.2$ & 0.02 \\
Motility $(\%)$ & $167.9 \pm 166.6$ & $160.3 \pm 116.8$ & 0.79 \\
$\begin{array}{l}\text { Total sperm count }(\times 106) \\
\begin{array}{l}\text { Total motile sperm count } \\
(\times 106)\end{array}\end{array} \quad 91.6 \pm 95.4$ & $80.6 \pm 60.9$ & 0.33 \\
$\begin{array}{l}\text { Concentration of } \\
\text { recovered sperm }(\times 106 / \mathrm{ml})\end{array}$ & $24.1 \pm 19.9$ & $18.1 \pm 15.3$ & 0.007 \\
\hline
\end{tabular}

Table 4 Lowest values of semen findings in pregnancy cycle

\begin{tabular}{lc}
$\begin{array}{l}\text { Lowest values of semen findings in pregnancy } \\
\text { cycle }\end{array}$ & 0.5 \\
\hline Volume $(\mathrm{ml})$ & 5 \\
Concentration $(\times 106 / \mathrm{ml})$ & 15 \\
Motility $(\%)$ & 10 \\
\hline Total sperm count $(\times 106)$ & 2 \\
\hline Total motile sperm count $(\times 106)$ & 0.3 \\
\hline Concentration of recovered sperm $(\times 106 / \mathrm{ml})$ &
\end{tabular}




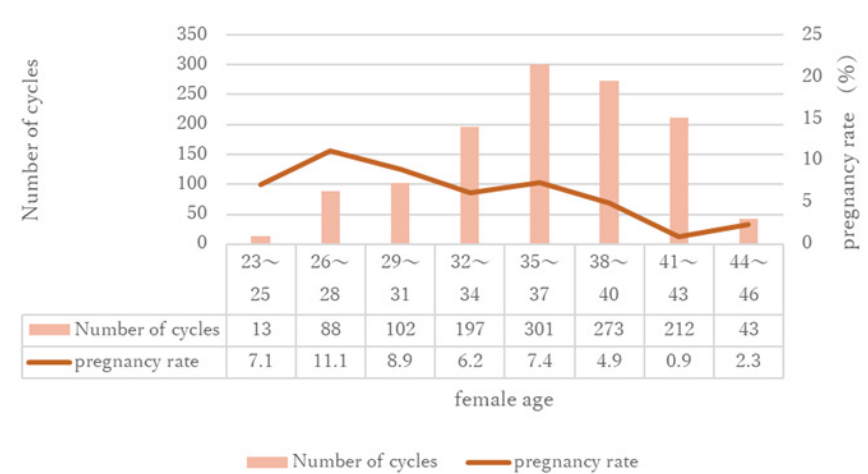

Figure I Number of IUI cycles and pregnancy rate by female age.

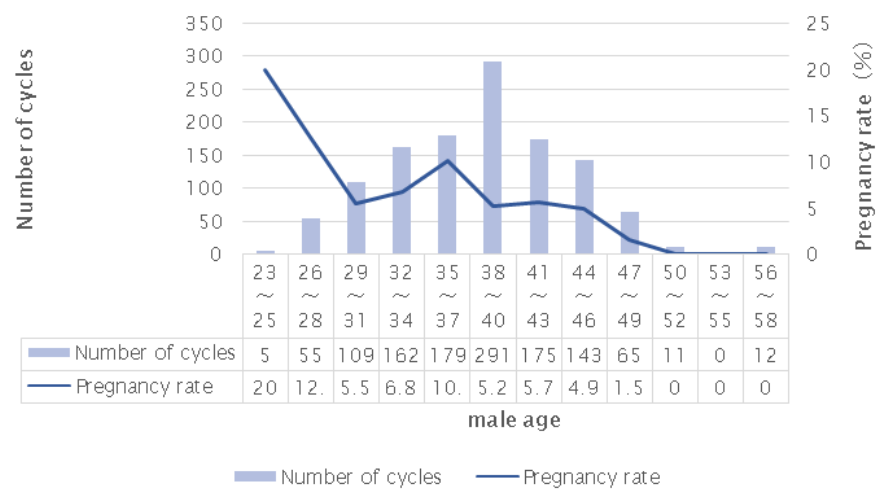

Figure 2 Number of IUI cycles and pregnancy rate by male age.
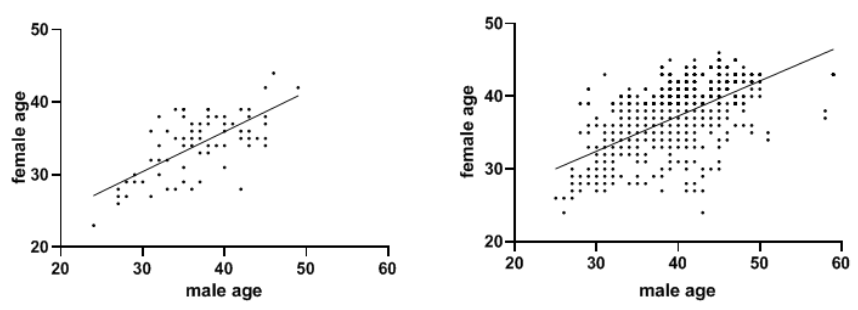

Figure 3 Age of couples in pregnant and non-pregnant cases.

\section{Pregnancy rate by number of IUI cycles}

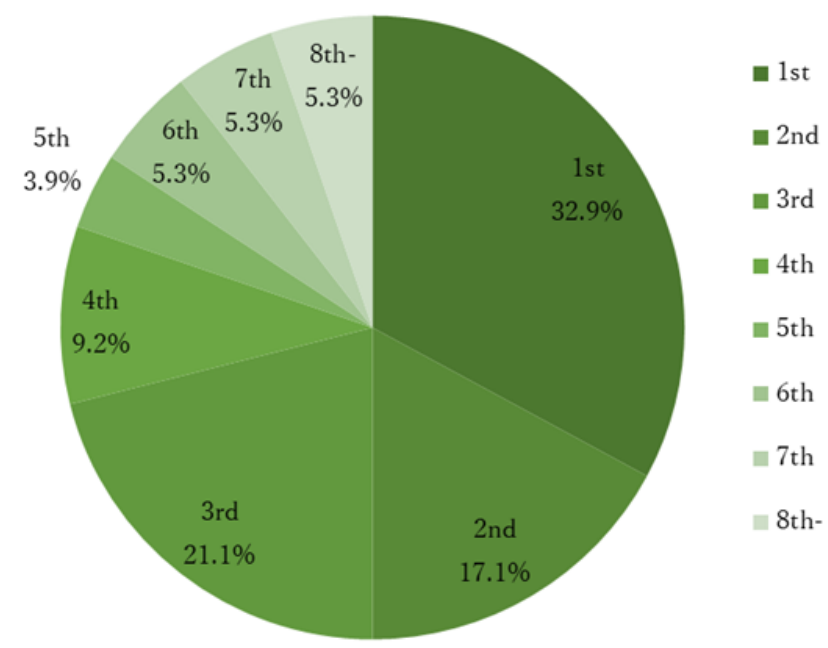

Figure 4 Pregnancy rate by number of IUI cycles.

\section{Discussion}

The pregnancy rate of $6.2 \%$ in our study is lower than the $5 \sim 20 \%{ }^{1,3,4}$ in previous national and international reports. This may be as a result of no age limit or frequency restriction in place for inseminations in our hospital, and several cases of women over 40 years old who repeated more than 10 cycles (maximum 21 cycles) were included. Additionally, the higher age of our patients and the fact that ovulation induction was not used to prevent multiple pregnancies in cases without ovulation disorders, may have contributed to a lower pregnancy rates. All pregnancies were singleton pregnancies. In assessing the number of inseminations and pregnancies, about $90 \%$ of the pregnancies were completed by the 6th cycle. In general, it is said that the cumulative pregnancy rate remains constant after five to six cycles and it does not increase beyond that; ${ }^{5}$ our institution proposed a step up to IVF for couples who had not become pregnant after the 5 th or 6 th cycle. Although there were eight pregnancies after the 7 th cycle $(4,1$ each at the 7th, 10th, 11th, 12th, and 14th cycle), IUI should ideally only be continued after the 7th cycle in patients who do not wish to undergo IVF. However, in women aged 38-39 years, successful insemination was commonly achieved at the first or second cycle, and for women aged 40 years and older the success rate leveled off after the first cycle; ${ }^{6}$ therefore, further attempts at IUI are not recommended for these age groups.

When considering the age at which insemination should be undertaken, it is often reported that the age of the woman has the greatest influence in determining the success of IUI. It is commonly believed that the younger the woman, the higher the pregnancy rate, and the age of the man is not associated with the pregnancy rate, with the age of the woman being the only factor affecting pregnancy. ${ }^{8}$ In this study, the pregnancy rate gradually declined with age for women, but there was no significant decline in the pregnancy rate for men until 46 years of age. The ages of the married couples correlates with the pregnancy rate as shown in Fig. 3, and it is difficult to determine an appropriate standard based on the ages of the woman and man alone. However, this study suggests that it is necessary to propose an immediate step-up to IVF, and to reconsider the continuation of IUI in women aged 41 years or older, and in men aged 47 years or older, as the pregnancy rate is markedly reduced at these ages. Several studies of significance have been reported. The best predictor of the pregnancy rate is total motile sperm count; ${ }^{2}$ pregnancy rates decrease significantly when the total sperm count is less than 10 million, ${ }^{9}$ and the pregnancy rate increases significantly when the total motile sperm count is greater than 20 million. ${ }^{10}$ Our study found significant differences in motility and concentration of recovered sperm. With regards to motility, previous studies have also reported that it is an indicator of pregnancy rate. ${ }^{11}$ A report from another institution, that performs sperm conditioning similar to our method of concentrating recovered sperm after adjustment, showed a significant difference in the concentration of sperm after adjustment, and the total motile sperm count. ${ }^{12}$ This suggests that semen findings, following adjustment by the density gradient method, may be an indicator of pregnancy. In addition, this study suggests that pregnancy by IUI is not expected when the total motile sperm count is less than 2 million, and the adjusted recovered sperm concentration is less than $300,000 / \mathrm{ml}$.

\section{Conclusion}

The pregnancy rate for IUI at our hospital is $6.2 \%$ per cycle, which is lower than national and international standards. Our study has determined that two main factors contribute to this: the individual 
ages of the couples and the quality of the semen. The motility and the concentration of recovered sperm seemed to be a possible determinant for the pregnancy rate. Both male and female ages contribute to successful outcomes, with implications that women over 41 years old and men over 47 should be encouraged to enroll for IVF. In summary, it is important to make a comprehensive decision by taking into account the semen analysis results and the couple's ages when determining the number of IUIs for better outcomes. There is further scope for research as our institution does not give any fertility treatment for women who have a regular ovulation cycle naturally. The addition of this service could result in a different pregnancy rate for our hospital.

\section{Acknowledgments}

None.

\section{Conflicts of interest}

The author declares there is no conflict of interest.

\section{Funding}

None.

\section{References}

1. Luka Dinelli, Blandine Courbiere, Vincent Achard, et al. Prognosis factors of pregnancy after intrauterine insemination with the husband's sperm: conclusions of an analysis of 2,019 cycles. Fertil Steril. 2014;101(4):994-1000.

2. Willem O, Nathalie D, Annelies T. Semen quality and prediction of IUI success in male subfertility: a systematic review. Repro BioMed Online. 2014;28(3):300-309.

3. Hakan E Duran, Mahmood Morshedi, Thinus Kruger, et al. Intrauterine insemination: a systematic review on determinants of success. Hum Reprod Update. 2002;8(4):373-384.
4. Tetsuro Honda, Mai Tsutsumi, Fumio Komoda, et al. Acceptable pregnancy rate of unstimulated intrauterine insemination: a retrospective analysis of 17,830 cycles. Reprod Med Biol. 2015;14:27-32.

5. Richard P Dickey, Steven N Taylor, Peter Y Lu, et al. Effect of diagnosis, age, sperm quality, and number of preovulatory follicles on the outcome of multiple cycles of clomiphene citrate-intrauterine insemination. Fertil Steril. 2002;78(5):1088-1095.

6. Isiah D Harris, Stacey A Missmer, Mark D Hornstein. Poor success of gonadotropin-induced controlled ovarian hyperstimulation and intrauterine insemination for older women. Fertil Steril. 2010;94(1):144 148.

7. Pieternel Steures, Jan Willem van der Steeg, Ben WJ Mol, et al. Prediction of an ongoing pregnancy after intrauterine insemination. Fertil Steril. 2004;82(1):45-51.

8. Takayuki Tatsumi, Eri Ishida, Kuniko Tatsumi, et al. Advanced paternal age alone does not adversely affect pregnancy or live-birth rates or sperm parameters following intrauterine insemination. Reprod Med Biol. 2018;17(4):459-465.

9. BJ Van Voorhis, M Barnett, AE Sparks, et al. Effect of the total motile sperm count on the efficacy and cost-effectiveness of intrauterine insemination and in vitro fertilization. Fertil Steril. 2001;75(4):661-668.

10. JG Brasch, R Rawlins, S Tarchala, et al. The relationship between total motile sperm count and the success of intrauterine insemination. Fertil Steril. 1994;62(1):150-154.

11. Uranchimeg, Dorjprev, Kuwahara A, et al. Effect of semen characteristics on pregnancy rate following intrauterine insemination. $J$ Med Invest. 2011;58(1-2):127-133.

12. Yumiko Okada, Akihiko Sekizawa, Hidekazu Saitoh, et al. Prognostic Factors of Pregnancy after Intrauterine Insemination with Husband's Semen. J Fertilization Implantation. 2017;34(1):58-64. 